\title{
A Characterization of Resource Allocation in LTE Systems Aimed at Game Theoretical Approaches
}

\author{
Luca Anchora*, Luca Canzian ${ }^{\dagger}$, Leonardo Badia*£, Michele Zorzi ${ }^{\dagger \S}$ \\ * IMT Institute for Advanced Studies, piazza S. Ponziano 6, 55100 Lucca, Italy \\ $\dagger$ Dipartimento di Ingegneria dell'Informazione (DEI), University of Padova, via Gradenigo 6B, 35131 Padova, Italy \\ $\S$ Consorzio Ferrara Ricerche (CFR), via Saragat 1, blocco B, 44122 Ferrara, Italy \\ e-mail: luca.anchora@imtlucca.it,canzian@dei.unipd.it,l.badia@imtlucca.it,zorzi@dei.unipd.it
}

\begin{abstract}
This paper proposes a novel approach, based on game theory, for radio resource allocation in the downlink of cellular networks using Orthogonal Frequency Division Multiple Access. The reference technology is the Long Term Evolution of the 3GPP UTRAN. The main contribution is to identify a model for the allocation objectives, and how to approach them in a tunable manner. The resource management issue is framed in the context of spectrum sharing, where multiple entities agree on utilizing the radio access channel simultaneously. A trade-off between sum-rate throughput and fairness among the users is identified and addressed through game theory, i.e., moving the operation of the system towards a stable Pareto efficient point. Such a methodology can be implemented with low complexity while ensuring logical modularity of the overall system. Numerical results are also shown, to exemplify the validity of the proposed approach.
\end{abstract}

Index Terms-Radio resource management, cellular networks, radio communication, game theory.

\section{INTRODUCTION}

Cellular wireless systems have been able to improve their transmission rates, so as to reach "high speed" communication, thanks to the introduction of channel-aware radio resource allocation. This means that packet scheduling and the corresponding assignment of physical layer resources are dynamically performed according to the channel conditions and Quality of Service (QoS) experienced by the users.

An important scenario where this principle finds application is represented by the Long Term Evolution (LTE) of Third Generation $(3 \mathrm{G})$ systems [1]. In this technology, the multiple access scheme in the downlink uses Orthogonal Frequency Division Multiple Access (OFDMA). Such a technology exploits multiple orthogonal subcarriers which can be used to take advantage of multi-user diversity [2]. However, given the key role played by the physical layer and the correlation of channel quality, the principles of a fair scheduling of multiple users are difficult to harmonize with the efficient resource allocation aiming at maximizing throughput.

In this paper, along the lines of [3], we utilize a modular representation of the radio resource management procedure which is split between two functional entities, i.e., a creditbased scheduler and the actual resource allocator, operating at the transport layer and the medium access layer, respectively. The scheduler determines which packets, taken from different flows, are candidates to be served in the next allocation round. The resource allocator associates the packets with groups of

This work was supported by the FP7 EU project SAPHYRE, grant agreement no. 248001 .
OFDMA subcarriers, also accessed in a Time Division (TD) fashion, so that the resources to allocate are time/frequency resource blocks. In this choice, the resource allocator exploits a degree of freedom, represented by the number of packets selected by the scheduler (larger than the number of slots).

The resulting allocation can be regulated according to a trade-off between two contrasting objectives, i.e., that of throughput maximization, which is achieved by selecting the packets only according to a channel quality rationale, and fairness among the flows, which requires to pursue equity among the achieved rates. Indeed, this trade-off is reflected by the number of packets selected by the scheduler: when it is minimum, i.e., only the packets that fit the OFDMA frame are selected, all packets are mandatorily allocated, and the resource allocator has no choice. Here the allocation is only determined by the credit-based scheduler, which ensures fairness (the users with higher credits are allocated). Conversely, if the number of selected packets is high, the resource allocator can restrict the selection to the packets of the users with the best quality, entirely neglecting any fairness among flows.

Therefore, to solve the trade-off we present an original approach based on game theory which tries to combine both objectives in an efficient yet easy to implement manner. The key idea is to treat them as two players of a non-cooperative game. The resulting Nash equilibria are considered as possible solutions to the radio management problem, which exhibit a low computational cost, yet, under certain conditions, satisfactory performance. After discussing the proposed approach and its possible implementation, we also present some simple numerical evaluations for a two-person game which confirm the goodness of our approach and its ability to regulate the trade-off in a Pareto efficient allocation point.

The outline of the rest of the paper is as follows. Section II outlines related approaches presented in the literature. Section III describes the properties of the LTE technology and discusses the layered characterization we gave to the resource allocation procedure. Section IV introduces our proposal, whose rationale is based on game theory, which is used to determine a trade-off between throughput efficiency and fairness among the users. Supporting numerical results are shown in Section $\mathrm{V}$ and we conclude in Section VI.

\section{StATE OF THE ART}

Adaptive multi-user multi-carrier allocation schemes based on instantaneous Channel State Information (CSI) in OFDMA systems allow significant performance improvements in terms 
of allocation efficiency. This happens thanks to the exploitation of the multiuser diversity principle, where subcarriers are preferably assigned to users experiencing favorable subchannel conditions and higher order modulation can be used to transport more bits per OFDMA symbol.

In this section we focus on the resource allocation optimization problem in OFDMA downlink systems with perfect CSI at the base station. In the literature there is no unique formulation for this type of problem. The most common formulation is the weighted sum rate maximization subject to some transmit-power constraints. For any fixed subchannel assignment, the optimal solution is achieved by multilevel waterfilling [4] for the continuous rate case (channel capacity is considered) and greedy or bisection allocation algorithms [5] for the integer-bit constellation case (bit rate constrained to real modulation schemes). When equal weights are considered, the optimal subchannel assignment is simply obtained by giving each subchannel to the user with the best gain to noise ratio [4]. This is called the max-sum-capacity rule, which results in the most efficient use of the resources in terms of throughput but can lead to unfairness and instability, especially for non-symmetrical channel conditions and nonuniform traffic patterns [2]. However, in the general case, finding the optimal subchannel assignment is a combinatorial problem whose complexity increases exponentially with the number of subcarriers. To find an efficient suboptimal algorithm, [4] considers a convex relaxation method, allowing time sharing in each subchannel. In this way the problem becomes convex and can be solved in polynomial time using interior-point methods. A further reduction in computational complexity is achieved considering a constant power for the used subchannels. In [6] a solution of the problem is efficiently computed using Lagrange dual decomposition and considering that the duality gap is zero when the number of subcarriers tends to infinity. Previously described works consider continuous rate adaptation. An additional constraint is added in [1] taking into account that real communication systems rely on integer-bit constellations. Moreover, since LTE is considered, the modulation and coding scheme for a given user has been considered fixed during a scheduling period. Also in this case the problem is combinatorial and a sub-optimal algorithm has been designed to reduce the computational complexity.

Another way of tackling the problem is power minimization subject to rate constraints for each user. In [6], similar to the weighted sum rate maximization, the Lagrange dual decomposition method has been proposed. In [7] an integerbit constellation is considered and the power has been assumed to be a convex and increasing function of the bit rate (most popular coding and modulation schemes satisfy this condition). Due to the combinatorial nature of the problem, a convex relaxation has been used to obtain a sub-optimal solution.

Another approach is proposed in [8] where a fairness constraint is taken into account: the smallest capacity among all users is maximized, subject to a total transmit-power constraint. Variable bit rate traffic is considered, but the formulation can be slightly modified to consider constant bit rate traffic. This objective function can lead to inefficiencies if some users experience deeply faded subchannels. In [2], in order to support delay-sensitive applications, an approach that maximizes the total utility with respect to mean queue delays is proposed. Also in these last works, suboptimal solutions are computed due to the combinatorial nature of the problem. Finally, we cite the Proportional Fair scheduling [2], that aims at maximizing the logarithm of the average data rates to trade off spectrum efficiency and fairness among users.

To sum up, it is difficult to formulate the desired optimization goal and constraints for the multi-user multi-carrier allocation problem, in particular when mixed traffic with different QoS requirements is considered. Also, the set selection nature of the sub-carrier allocation leads to a combinatorial problem that requires an exhaustive search, with exponentially increasing complexity. Simplified approaches must be considered to design real time algorithms exploiting instantaneous subchannel information. This motivates us to consider an approach that does not claim optimality with respect to a subjective utility function, but rather is computationally lightweight and able to find a good trade-off between aggregate performance (in terms of throughput/spectrum efficiency) and fairness among flows.

\section{OVERVIEW OF LTE AND SYSTEM MODEL}

LTE is a set of improvements to the Universal Mobile Telecommunications System (UMTS) introduced in the 3rd Generation Partnership Project (3GPP) Release 8 [9]. It represents efficient packet-based radio access networks allowing high throughput, low latency and low operating costs. Small enhancements have been introduced on LTE specifications in Release 9 [10]. The next step for LTE evolution is LTE Advanced which is currently being standardized in Release 10 [11], the major candidate technology for the so-called International Mobile Telecommunications (IMT)-Advanced.

Rel-8 LTE adopts OFDMA in the downlink for its robustness against multipath interference and to allow a high spectral efficiency exploiting time and frequency dependent scheduling and Multiple Input Multiple Output (MIMO) techniques. In the uplink, in order to maintain user orthogonality in the frequency domain, a Single Carrier Frequency Division Multiple Access (SC-FDMA) is adopted. Rel-8 LTE supports both Frequency Division Duplexing (FDD) and Time Division Duplexing (TDD) and uses multiple transmission bandwidths (i.e., 1.4, 3, 5, 10, 15 and $20 \mathrm{MHz}$ ) and multiple modulation schemes (i.e., QPSK, 16QAM and 64QAM) allowing peak rates of 300 $\mathrm{Mb} / \mathrm{s}$ in downlink and $75 \mathrm{Mb} / \mathrm{s}$ in uplink.

We consider now the scheduling degree of freedom for the downlink of Rel-8 LTE. The basic unit of resource is the Resource Block (RB), which is made of 12 adjacent subcarriers (15 kHz of subcarrier spacing) and has a duration of $0.5 \mathrm{~ms}$ (one slot), which correspond to 6 or 7 OFDM symbols depending on the cyclic prefix length chosen $(4.7$ $\mu \mathrm{s}$ or $16.7 \mu \mathrm{s})$. The Scheduling Block (SB) is the smallest resource unit that the scheduler can assign. It is made of two consecutive RBs, and therefore has a duration of $1 \mathrm{~ms}$ (one subframe). During the duration of a scheduling period, which is equal to the duration of a SB (i.e., $1 \mathrm{~ms}$ ), the Modulation and Coding Scheme (MCS) must be fixed for each user in the non MIMO configuration. For the MIMO configuration, a maximum of two different MCSs can be used for data belonging to two different transport blocks [1]. 
LTE Advanced is a further evolution of LTE Release 8 and 9 which is supposed to meet the requirements for IMTAdvanced and enhance them to future operator and user needs. It shall support a wider transmission bandwidth using both contiguous and non-contiguous carrier aggregation, achieving flexible spectrum usage while maintaining backward compatibility with Rel-8. Moreover, it shall enhance multiantenna and Coordinated Multi-Point transmission/reception techniques. These improvements are expected to allow peak rates of $1 \mathrm{~Gb} / \mathrm{s}$ in downlink and $500 \mathrm{Mb} / \mathrm{s}$ in uplink.

Different radio resource management strategies are required for organizing and bringing together multiple users and letting them receive data in an LTE system (note: we are considering the downlink, which is the only direction using OFDMA multiplexing). In particular, multiple flows directed to the users are to be coordinated, so that a number of packets are selected for possible transmission from each flow. In the following, this operation will be referred to as scheduling. However, according to the above discussion, actual transmission requires to match the selected packets to a given resource block in a channel-aware fashion. Thus, it is necessary to eventually select which resources to utilize for the selected packets. Such an operation will be referred to as resource allocation.

The design of policies for resource management is intentionally left open in the standards to allow developers to implement their own strategy of choice. However, in the following we adopt a two-fold model where scheduling and resource allocation are managed by two different modules: a scheduler, operating at the transport layer (thereby possibly distinguishing among different kinds of traffic) and a resource allocator, which actually implements the Medium Access Control (MAC) sublayer. The scheduler determines which packets must be passed to the allocator and their order according to an internal scheduling policy. The allocator selects for transmission a subset of them with the aim of maximizing the advantages of multiuser diversity. In this case only a loose cross-layer is introduced, guaranteeing a certain modularity between scheduler and radio resource allocator (RRA).

In particular, we call $L$ the number of resource blocks that the resource allocator is entitled to assign. This is subject to a constraint $L \leq L_{\max }$, where $L_{\max }$ is a maximum value which corresponds to assigning every resource block. For simplicity, we consider that, to limit the interference caused to the neighboring cells, $L$ is set to a fixed value which is less than or equal to $L_{\max }$. The value assigned to $L$ is communicated to the scheduler by the resource allocator. Actually, this represents a form of cross-layer interaction among the modules, which is intentionally kept to a minimum level, thereby promoting modularity and tunability of the approach.

Upon knowing $L$, the scheduler determines a number $D$ of packets to send to the resource allocator, where in general $D \geq$ $L$. The exact choice of $D$ influences the entire allocation. As a matter of fact, if $D=L$, the resource allocator has no degree of freedom as to which packets to allocate (while, obviously, it must allocate the packets to the best channels as perceived by the users). By increasing $D$, the resource allocator can achieve a higher throughput by selecting only $L$ packets out of $D$, according to a channel-aware policy, although at the price of a possibly decreased fairness.

\begin{tabular}{lc|c|c|c|c|} 
& & \multicolumn{4}{c}{ Resource Allocator } \\
& & \multicolumn{1}{c}{$L$} & \multicolumn{1}{c}{$L+1$} & $\ldots$ & $2 L$ \\
\cline { 3 - 6 } Scheduler & $L$ & $1, T_{\min }$ & 0,0 & 0,0 & 0,0 \\
\cline { 3 - 6 } & $L+1$ & 0,0 & $\ldots$ & 0,0 & 0,0 \\
\cline { 3 - 6 } & $\ldots$ & 0,0 & 0,0 & $\ldots$ & 0,0 \\
\cline { 3 - 6 } & $2 L$ & 0,0 & 0,0 & 0,0 & $\frac{1}{2}, T_{\max }$ \\
\cline { 3 - 6 } & & & & &
\end{tabular}

Fig. 1. Bi-matrix representation of the game

\section{The Proposed Game-Theoretic Approach}

The choice of $D$ determines a trade-off between the possible objectives of throughput and fairness. We now present a gametheoretic approach to set $D$; we remark that the main point of our discussion does not lie in optimizing the performance of the resulting algorithm, which is left for future research. Rather, our proposed methodology enables a dynamic setup of $D$ without any need for a preliminary evaluation, e.g., where $D$ is set to some arbitrary value, of the possible equilibria of the system, nor it is required to re-compute the system equilibria if the network and channel conditions change. Instead, the choice of $D$ is directly derived from the definitions of the contrasting utilities between which a tradeoff is sought (specifically, throughput and fairness). Together with the separation of the resource management process into two functional entities (scheduler and RRA), this is key to achieve a computationally efficient online allocation strategy.

In our formulation, the scheduler (player 1) and the RRA (player 2) are represented as players of a game whose aim is the decision of the value for $D$. Both players make a proposal $s_{j}$, with $j=1,2$, respectively. The idea is that, if proposals $s_{1}$ and $s_{2}$ coincide, $D$ is selected as their common value. However, the choice of $s_{1}$ and $s_{2}$ is also done according to the utility of the proposer, i.e., the fairness for the scheduler and the throughput for the RRA, respectively.

In the following, we introduce some assumptions for the sake of simplicity in the exposition. We consider a network scenario with only two users; this is not to be confused with the two "virtual" players of the game, i.e., the scheduler and the resource allocator. Besides, this assumption is just made for ease of implementation in the simulator, but can be relaxed quite naturally to scenarios with $N>2$ users. We model the system as a static game in normal form, as follows:

- the players are the scheduler and the RRA.

- their action spaces are the set of values of $D$ that can be proposed, i.e. $S_{1}=S_{2}=\{L, L+1, \ldots, 2 L\}$.

- both payoffs are 0 if the proposals $s_{1}$ and $s_{2}$ do not coincide, i.e., there is no agreement on the value of $D$.

- when $s_{1}=s_{2}$, the payoffs are assigned to fairness $F\left(s_{1}, s_{2}\right)$ for the scheduler, measured using Jain's index [12], and the throughput $T\left(s_{1}, s_{2}\right)$ for the RRA.

The last point is arbitrary, as other definitions can be used; the important requirement is that $F(s, s)$ and $T(s, s)$ are decreasing and increasing in $s$, respectively. The resulting bimatrix representation of the game is given in Fig. 1. The fairness is a decreasing function of $D$ : its maximum value is 1 while the minimum is $1 / 2$ (i.e., $1 / N$ where $N$ is the number of flows). On the other hand, the throughput is an 
increasing function of $D$ varying in the range $\left[T_{\min }, T_{\max }\right]$, where $T_{\min }$ is achieved when no degree of freedom is given to the allocator, while $T_{\max }$ is obtained when the RRA has enough freedom to allocate only the best $L$ resources. Both maximum throughput and minimum fairness are reached for $D=2 L$, under the assumption that there are always at least $L$ packets available for selection by the scheduler from each queue. All the strategies along the diagonal are Pareto efficient Nash equilibria. This means that improving the payoff of one player results in worsening the other's outcome. Thus, once the value of $L$ is fixed, there is no unique evolution of the game and, in any case, a trade-off is encountered.

To determine a trade-off point, we propose an algorithm which tries to automatically estimate an efficient value of $D$ for each frame. The value is chosen considering the entire history of the game, thus the model we propose is a repeated game with perfect information. The aim is to reach an acceptable level for both payoffs after a number of repetitions. Note that this proposed algorithm is just an example and can be replaced by other analogous procedures.

1) Both scheduler and RRA randomly pick a value for $D$.

2) If the choices coincide, $D$ is set and the game ends, otherwise a bargaining phase goes on until a common point is chosen. Every time the players disagree, both get zero payoff. 3) The goal of each round of the loop is moving towards the diagonal of the bi-matrix step-by-step. Each player decides whether or not to change its previous proposal based on its level of satisfaction (i.e., the ratio between the value actually achieved and the maximum achievable). The higher the satisfaction, the higher the probability that a player changes its choice with a value more convenient for the other. If $S \_D$ and $R R A_{-} D$ are the proposals for $D$ made by the scheduler and the allocator, respectively, and $S \_s$ and $R R A \_s$ the respective levels of satisfaction when the game is played, we select the changes as follows.

- If $S \_D>R R A \_D$, we are in the lower triangle of the matrix. We can move towards the diagonal by going up (decrement of $S_{-} D$ ), or right (increment of $R R A_{-} D$ ), or in both directions. For both players, these options lead to higher values in their own utility function to the detriment of the other's, thus the willingness to change should be a decreasing function of the respective satisfaction level. Thus, we select

$$
\begin{aligned}
\operatorname{Prob}\left\{S \_D \text { up }\right\} & =1-S_{-} s \\
\operatorname{Prob}\left\{R R A \_D \text { right }\right\} & =1-R R A \_s
\end{aligned}
$$

- If $S \_D<R R A \_D$, we are in the upper triangle of the matrix. The diagonal can be reached by going down $\left(S \_D\right.$ increment), or left (RRA_D decrement), or in both directions. The situation is now reversed, as a deviation in its own action implies a reduction in the payoff of each player in favor of the other's. Therefore, the probability of moving must be an increasing function of the respective satisfaction, which is obtained for example by choosing

$$
\begin{aligned}
\operatorname{Prob}\left\{S \_D \text { down }\right\} & =S \_s \\
\operatorname{Prob}\left\{R R A \_D \text { left }\right\} & =R R A \_s
\end{aligned}
$$

In this manner, we define an algorithm whose goal is to lead the choice of $D$ towards an intermediate value which offers both good throughput and satisfactory fairness.

\section{NUMERICAL RESULTS}

We ran evaluations within a simple LTE simulator to verify the ability of the proposed approach to converge towards a trade-off among the payoff functions of the two players. All the performance indices are characterized by a confidence interval of $95 \%$ with a maximum relative error of $5 \%$.

We developed and used a simple asynchronous event-driven simulator, written in $\mathrm{C}++$, which reproduces a base station transmitting to two different mobile users. The base station contains a packet scheduler with two queues (one for each user) and an RRA module. The scheduler is credit-based and tries to guarantee fairness by selecting packets from the queues according to their residual credit. Flows are assumed to have always backlogged traffic. The RRA manages the resource allocation according to a greedy criterion: slots and packets are matched in order to maximize the total throughput given the channel condition of each user, which are assumed to be independent of each other.

The radio channel model represents each frequency subchannel by means of a two-state Markov channel (GilbertElliot model) whose state is updated after each time slot to take into account channel correlation over time. The number of subcarrier groups is 16 while the time slots for each frame are 24 , for a total of 384 resource blocks. A different average noise power is associated with each of the two states of the chain, thus different values of capacity can be reached (according to the Shannon formula). For simplicity, when the Gilbert-Elliot channel is in the good state, interference and noise power are treated as a random variable with uniform distribution between 1 and $2 \mathrm{~mW}$; similarly, in case of bad channel, the interference plus noise power is uniformly distributed between 1 and 200 $\mathrm{mW}$. The transmission power per slot is fixed to $1 \mathrm{~mW}$. The main system parameters are summarized in Table I.

In Fig. 2 and Fig. 3 the fairness and the normalized throughput as a function of time are shown for several values of $D$ when $L=300$ packets. They confirm what was expected from our analysis: the fairness is a decreasing function of $D$ while the throughput increases. When $D=L$, we have that the fairness is always 1, the maximum value according to Jain's index. On the other hand, the normalized throughput has its minimum value because the resource allocator has no freedom in the choice of the packets to transmit and the user diversity cannot be exploited.

When $D$ is increased, the two performance indices considered have contrasting behaviors, as already expressed in the previous section: the fairness undergoes a decrease while the throughput starts increasing. The introduction of a certain

\begin{tabular}{|c|c|}
\hline Parameter & value \\
\hline number of flows & 2 \\
\hline packet size & 500 bytes \\
\hline $\operatorname{Pr}\{G O O D \rightarrow G O O D\}$ & 0.9 \\
\hline $\operatorname{Pr}\{B A D \rightarrow B A D\}$ & 0.8 \\
\hline number of subcarriers & 16 \\
\hline time slots per frame & 24 \\
\hline frame duration & $1 \mathrm{~ms}$ \\
\hline transmission power per slot & $1 \mathrm{~mW}$ \\
\hline
\end{tabular}

TABLE I

MAIN SYSTEM PARAMETERS 


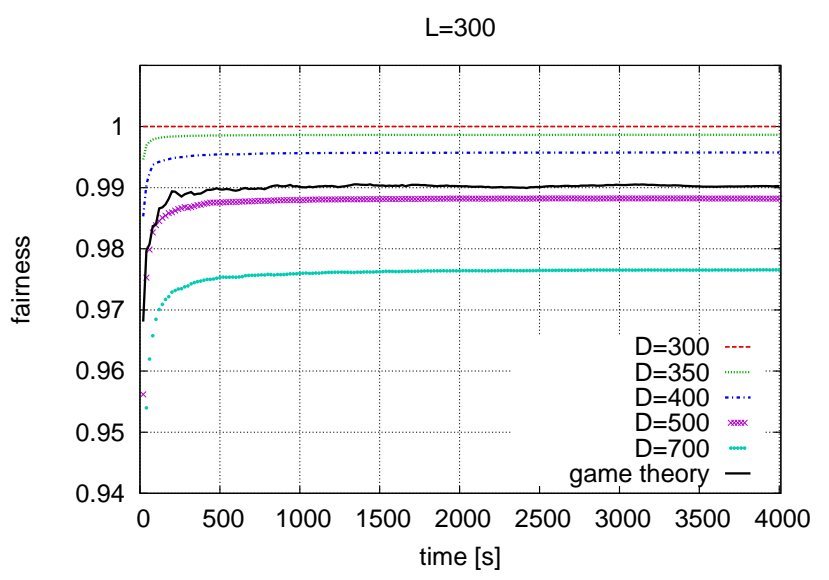

Fig. 2. Fairness over time for different values of $D$.

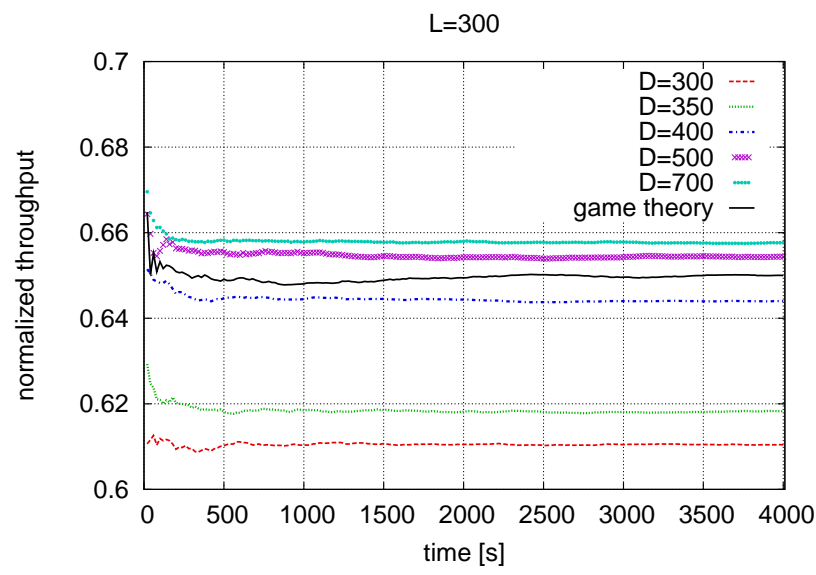

Fig. 3. Throughput over time for different values of $D$.

freedom in the allocation choice shows its effects and the trade-off among the payoff of the two players becomes evident. Figure 4 clearly shows this situation: the points along the curve are the Pareto solutions of the game, one for each value of $D$, and there is no possibility to reach a better solution for one player without worsening the other's one.

All figures report the outcome of the game theoretic algorithm. Both in Fig. 2 and in Fig. 3, the automatic choice of $D$ leads to an intermediate value of both performance indices. This means that each player slightly reduces its own payoff for the sake of a better joint solution. In Fig. 4 it is shown that this new operating point is localized close to the Pareto boundary. Moreover, the proposed algorithm is quite simple and the convergence to a common value of $D$ is extremely fast, thus it is suitable for an online implementation. Indeed, in Figs. 2-3 the warm-up period is quite short, about $300 \mathrm{~ms}$.

For completeness, we ran other tests by varying $L$ in the range $[100,350]$. In all these cases we obtained that the fairness increased with the value of $D$ while the throughput decreased. The operation point reached by the proposed algorithm always approximately lies on the Pareto boundary.

\section{CONClusions}

In this paper we have presented a novel design approach for resource management in OFDMA/TDMA cellular networks

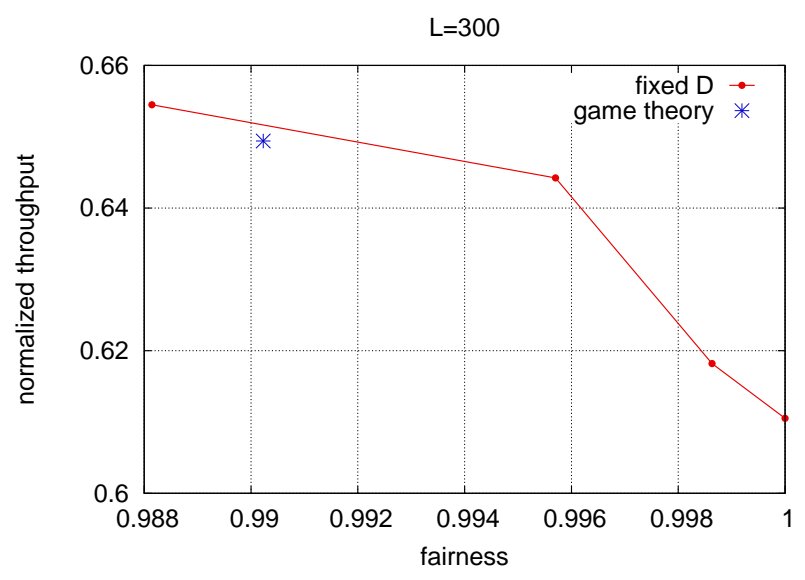

Fig. 4. Pareto boundary and operating point of the algorithm.

such as LTE. A cross-layer approach has been explored, where scheduler and radio resource allocator exchange a limited amount of information to provide both an adequate level of fairness among flows and a high throughput. A game theoretic model of the system has been proposed and a feasible algorithm for the dynamic setting of a system parameter has been evaluated. The results obtained through simulation show that the proposed solution is able to trade-off fairness requirements and throughput.

Possible future works include the extension to a multicellular network, where several base stations coexist and share resources trying to minimize mutual interference through a proper resource allocation. Moreover, we plan to implement the proposed approach in a more detailed network simulator.

\section{REFERENCES}

[1] R. Kwan, C. Leung, and J. Zhang, "Resource allocation in an LTE cellular communication system," in Proc. IEEE ICC, Dresden, Jun. 2009.

[2] G. Song, Y. Li, and L. J. Cimini, "Joint channel- and queue-aware scheduling for multiuser diversity in wireless OFDMA networks," IEEE Trans. Commun., vol. 57, no. 7, pp. 2109-2121, Jul. 2009.

[3] L. Badia, A. Baiocchi, A. Todini, S. Merlin, S. Pupolin, A. Zanella, and M. Zorzi, "On the impact of physical layer awareness on scheduling and resource allocation in broadband multicellular IEEE 802.16 systems communication system," IEEE Wireless Commun. Mag., vol. 14, no. 1, pp. 36-43, Feb. 2007.

[4] L. Hoo, B. Halder, J. Tellado, and J. Cioffi, "Multiuser transmit optimization for multicarrier broadcast channels: asymptotic FDMA capacity region and algorithms," IEEE Trans. Commun., vol. 52, no. 6, pp. 922930, Jun. 2004.

[5] B. Krongold, K. Ramchandran, and D. Jones, "Computationally efficient optimal power allocation algorithms for multicarrier communication systems," IEEE Trans. Commun., vol. 48, no. 1, pp. 23-27, Jan. 2000.

[6] K. Seong, M. Mohseni, and J. Cioffi, "Optimal resource allocation for OFDMA downlink systems," in IEEE International Symposium on Information Theory, Seattle, WA, Jul. 2006.

[7] C. Y. Wong, R. Cheng, K. Letaief, and R. Murch, "Multiuser OFDM with adaptive subcarrier, bit, and power allocation," IEEE J. Sel. Areas Commun., vol. 17, no. 10, pp. 1747-1758, Oct. 1999.

[8] W. Rhee and J. Cioffi, "Increase in capacity of multiuser OFDM system using dynamic subchannel allocation," in Proc. IEEE VTC, vol. 2, Tokyo, May 2000, pp. 1085-1089.

[9] LTE physical layer - general description, TS 36.201 (V8.3.0) ed., 3GPP, March 2009.

[10] LTE physical layer - general description, TS 36.201 (V9.1.0) ed., 3GPP, March 2010.

[11] Requirements for further advancements for Evolved Universal Terrestrial Radio Access (E-UTRA), TR 36.913 (V9.0.0) ed., 3GPP, Dec. 2009.

[12] R. Jain, D. Chiu, and W. Hawe, "A quantitative measure of fairness and discrimination for resource allocation in shared computer systems," DEC Research Report TR-301, 1984. 